\title{
Studies on the growth and the development of the Claw capsule in new born calves of different breeding lines using linear measurements
}

\begin{abstract}
This study evaluated claw development in 80 calves of different genetic background during the first 70 days of life. Linear claw measurements were found to be useful tools for this purpose. Large differences in claw parameters were observed, depending on the position of the claws, gender and breeding line, including interaction effects between gender and breeding line.

In all four pairs of claws, the medial claws were longer than the lateral claws. Claw width is significantly wider in lateral claws, both hind and fore. Male calves showed significantly higher values than females in most measured parameters except bulb length and angle of the bulb.

Comparing breeding lines, significant differences in some parameters (claw width, length of the diagonal, sole length, axial wall length) were found, which are in discussion to be important in claw soundness. Older cows produced calves with larger claws than calves of younger cows.
\end{abstract}

Key Words: claw development, new born calf, horn growth, linear measurement

\section{Zusammenfassung}

Titel der Arbeit: Studien zum Wachstum und der Entwicklung des Hornschuhs neugeborener Kälber verschiedener Rassen mit Hilfe linearer Messungen

Die vorliegende Arbeit bewertet die Klauenentwicklung von 80 Kälbern verschiedener Zuchtlinien während der ersten 70 Lebenstage. Als Instrument für diese Bewertung wurden lineare Messungen herangezogen. Zwischen den gemessenen Klauenparametern wurden große Unterschiede gefunden, die sich aus der Position der Klaue, dem Geschlecht, der Zuchtlinie und aus Effekten zwischen Geschlecht und Zuchtlinien ergaben.

An allen vier Gliedmaßen waren die medialen Klauen länger als die lateralen. Die Breite der Klauen ist an den Vorder- und Hintergliedmaßen an den lateralen Klauen signifikant größer als an den medialen. Männliche Kälber zeigten in fast allen Parametern signifikant höhere Werte als weibliche, Ausnahmen waren die Ballenlänge und die Winkelung des Ballens.

Im Vergleich verschiedener Zuchtlinien konnten signifikante Unterschiede in einigen Parametern festgestellt werden (Klauenbreite, Wanddiagonale, Sohlenlänge und axiale Wandlänge), die als wichtige Parameter zur Erhaltung der Klauengesundheit bewertet werden. Die Kälber älterer Kühe hatten deutlich "größere” Klauen, als die jüngerer Kühe.

Schlüsselwörter: Klauenentwicklung, Kälber, Hornwachstum, lineare Messungen

1. Introduction

A large number of effects on claw development is described in the literature (RUSSKE, 2001; MANSKE, 2002; HUBER, 2002). However, relative little information is available for the claw development in calves. Claw development can be expressed as shape measured linearly, volume and density of the contents of the capsula ungulae. It is not known at what age the claw reaches maturity. This is of economic importance regarding the timing of first calving and the possibility that overburdening immature claws may have long-term implications for claw health. 
There is inadequate information regarding the time scale of claw deterioration after the point of maturity. The purpose of this paper is to examine linear measurements of the claws in new born calves as a method to develop basic protocols in claw development. Claw measurements as auxiliary traits for selection and for structural claw soundness are described by several authors. Wall length of the dorsal wall, bulb depth and bulb length, claw width, length of the diagonal and the angle of the dorsal wall are common traits to describe claws (BAUMGARTNER, 1988; HUBER, 1983; REURINK and VAN ARENDONK, 1987; VERMUNT and GREENOUGH, 1995). Most of the former investigations have been performed in adult cattle being influenced by environmental or production stress over a long period of time (MEYER and GALBRAITH, 1998; LANDERER et al., 1998; LIVESEY et al., 1998) or in male beef cattle (NITSCHE, 1974; MÜLLER et al. 1975). A very extensive investigation is presented by RUSSKE (2001), based on 32 female heifers of the breeds German Holstein and German Brown Swiss.

This investigation describes the early development of the capsula ungulae in new born calves of different breeding lines under similar housing conditions. The objective of the study was to determine how early claw development occurs and whether there are differences within and among claws, genders or breeding lines.

Material and methods

\subsection{Study sample}

Beginning at day 4 post partum (p.p.), four investigations were performed at the Experimental Farm Oberschleissheim within a three weeks interval. Male and female calves of four breeding lines were measured under light sedation. These four breeding lines were the two-purpose breed German Fleckvieh (FV), the dairy breed German Holsteins (GH) and F1 crossbred calves of these two breeds. Crossbred calves with Fleckvieh sire and Holstein cow are assigned FV-GH, while crossbreds with Holstein sire and Fleckvieh cow are assigned GH-FV (Table 1).

Table 1

Number of calves within measurement, genetic origin and gender (Anzahl der Versuchstiere nach Messnummer, Abstammung und Geschlecht)

\begin{tabular}{lcccc}
\hline & $\begin{array}{c}\text { Measurement 1 } \\
(\sim 6 \text { days p.p. })\end{array}$ & $\begin{array}{c}\text { Measurement 2 } \\
(\sim 28 \text { days p.p. })\end{array}$ & $\begin{array}{c}\text { Measurement 3 } \\
(\sim 49 \text { days p.p. })\end{array}$ & $\begin{array}{c}\text { Measurement 4 } \\
(\sim 70 \text { days p.p. })\end{array}$ \\
\hline GH & 17 & 15 & 13 & 11 \\
FV & 20 & 17 & 16 & 14 \\
GH-FV & 24 & 24 & 21 & 21 \\
FV-GH & 24 & 21 & 20 & 31 \\
male & 41 & 35 & 34 & 33 \\
female & 44 & 42 & 36 & \\
\hline
\end{tabular}

The calves originate from the mating of $5 \mathrm{GH}$ sires and $9 \mathrm{FV}$ sires with totally $35 \mathrm{GH}$ and $31 \mathrm{FV}$ cows or heifers $\left(1^{\text {st }}\right.$ to $6^{\text {th }}$ lactation). Among all calves were 12 twins (from 6 twin births) and 3 calves originated from a triple birth. With exception of one twin birth, all multiple births originated from FV cows. ${ }^{1)}$

\footnotetext{
${ }^{1}$ All animals involved in this study were treated according to the guidelines for care and use of agricultural animals in research.

(Registration number of the Government of Upper Bavaria 211-2531.2-57/2000)
} 
Up to the $7^{\text {th }}$ day of life, all calves were fed individually colostrum and housed in single calf boxes on straw mats under outside conditions. From day 8, the calves were housed in groups on straw in a cold stall. They received - depending on age - milk replacer over an automatic milk feeder (Westfalia $\left.{ }^{\circledR}\right)$. In addition, they received ad libitum hay, corn (whole kernels) and on farm produced concentrate (pelleted).

The measurements were performed under light sedation with Xylazin 2\% (1.5 - 2.5 $\mathrm{ml} / 100 \mathrm{~kg}$ body weight). Body weight was recorded at each measurement day. All parameters were measured with a caliper ${ }^{1}$ and a goniometer ${ }^{2}$ being used in human physiotherapy.

The parameters studied are shown and defined in Figure 1.

WL - Wall length

BD - Bulb depth

BL - Bulb length

DIAG - Length of the diagonal

SL - Sole length

AWL - Axial wall length

CW - Claw width

ADW - Angle of the dorsal wall AB - Angle of the bulb (AB)

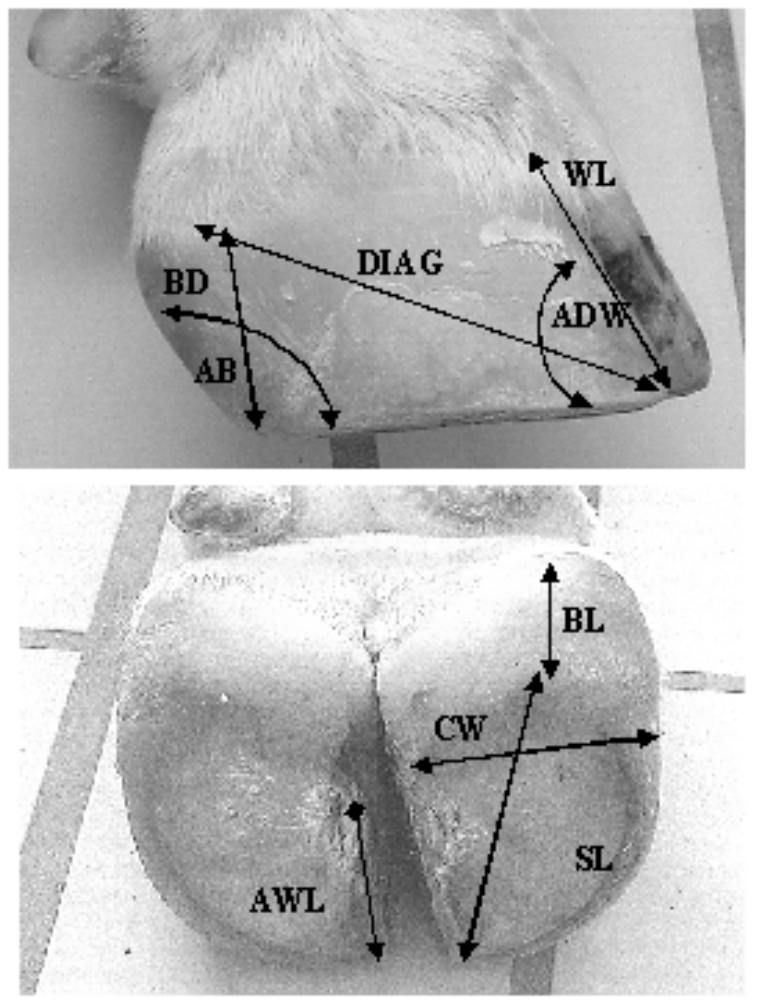

Length of the dorsal wall (distal border of the coronary band - toe) Height of the bulb, bulb coronet - vertical to the sole End of the loaded sole - end of the horny bulb Distance toe - bulb coronet Distance toe - end of the loaded sole

Toe - axial end of the weight-bearing border before slope

Distance between axial and abaxial surface taken at the widest point of the claw

Angle between dorsal wall and solear surface

Angle between bulb and solear surface

Fig. 1: Claw Parameters (Klauenparameter)

\subsection{Statistical procedure}

A variance analysis was performed using a General Linear Model (Proc GLM, SAS 8.1) with the following structure of fixed effects for all linear claw parameters (SAS/STAT, 1993):

\footnotetext{
${ }^{1}$ Schieblehre Mitutoyo, Artnr: A 61460

${ }^{2}$ Aktive Reha-Systeme FREI, Winkelmesser, Art.nr.: 4376200
} 


$$
\begin{aligned}
& \mathrm{Y}_{\mathrm{ijklmno}}=\mathrm{C}_{\mathrm{i}}+\mathrm{M}_{\mathrm{j}}+\mathrm{O}_{\mathrm{k}}+\mathrm{S}_{\mathrm{l}}+\mathrm{L}_{\mathrm{m}}+\mathrm{B}_{\mathrm{n}}+\left(\mathrm{O}_{\mathrm{k}} * \mathrm{~S}_{\mathrm{l}}\right)+\left(\mathrm{C}_{\mathrm{i}} * \mathrm{O}_{\mathrm{k}} * \mathrm{~S}_{\mathrm{l}}\right)+\mathrm{e}_{\mathrm{ijklmno}} \\
& \mathrm{Y}_{\mathrm{ijklmno}} \quad=\text { Observation } \\
& \mathrm{C}_{\mathrm{i}} \quad=\text { Claw }(\mathrm{i}=1-8) \\
& \mathrm{M}_{\mathrm{j}} \quad=\text { Number of Measurement }(\mathrm{j}=1-4) \\
& \mathrm{O}_{\mathrm{k}} \quad=\text { Genetic } \underline{\text { Origin }}(\mathrm{k}=1-4) \\
& \mathrm{S}_{\mathrm{l}} \quad=\underline{\operatorname{Sex}}(\mathrm{l}=1-2) \\
& \mathrm{L}_{\mathrm{m}} \quad=\text { Number of Lactation }(\mathrm{m}=1-6) \\
& \mathrm{B}_{\mathrm{n}} \quad=\text { Multiple Birth }(\mathrm{n}=1-2) \\
& \mathrm{O}_{\mathrm{k}} * \mathrm{~S}_{\mathrm{l}} \quad=\text { Interaction between Genetic } \underline{\text { Origin }} \text { and } \underline{\mathrm{S}} \mathrm{x} \\
& \mathrm{C}_{\mathrm{i}} * \mathrm{O}_{\mathrm{k}} * \mathrm{~S}_{\mathrm{l}}=\text { Interaction between } \underline{\text { Claw }} \text { and Genetic } \underline{\text { Origin }} \text { and } \underline{\mathrm{S}} \mathrm{x} \\
& \mathrm{e}_{\mathrm{ijklmno}} \quad=\text { Random Error }
\end{aligned}
$$

The following figures and tables contain (if not other stated) Least Squares Means (LSM) and corresponding Standard Errors of Estimation (SEE). The significance level was set for $\mathrm{p} \leq 0.05$.

\section{Results}

3.1. Relations between medial and lateral, fore and hind claws

Significant interactions were found for the parameters wall length, bulb depth, bulb length, diagonal, sole length, claw width and for the angles.

Wall length was longer in hind than in fore claws. In all four pairs of claws the medial claws are longer than the lateral claws. This is significant in hind left and both pairs of fore claws (Fig. 2).

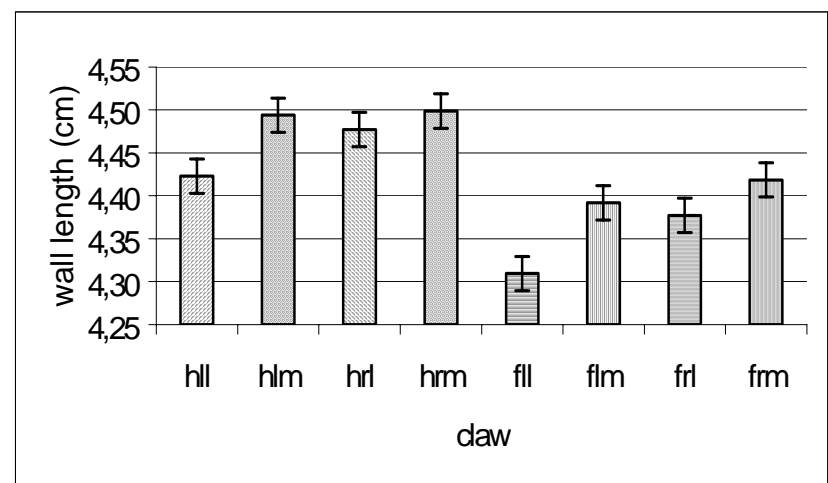

hll - hind left lateral / hind left medial / hrl - hind right lateral / hrm - hind right medial

fll - fore left lateral / flm - fore left medial / frl - fore right lateral / frm -fore right medial

Fig. 2: Differences in wall length (Unterschiede im Wachstum der Dorsalwand)

Bulb length and the diagonal are longer in medial than in lateral claws. The angle of the bulb is wider in medial claws. The angle of the bulb in all four hind claws is significantly wider than in the fore claws. That means, the hind claws are severely flatter than the fore claws (Fig. 3).

Bulb depth is larger in all fore claws than in hind claws. All lateral claws with exception of the hind left have a larger bulb depth than the medial ones. In addition, the lateral hind and fore claws are significantly wider than the medial claws. Comparing alone the medial or lateral claws, the fore claws are significantly wider 
than the hind claws. Comparing the medial and lateral claws, hind and fore pairs of claws are similar in the first three months of life (Fig. 4).

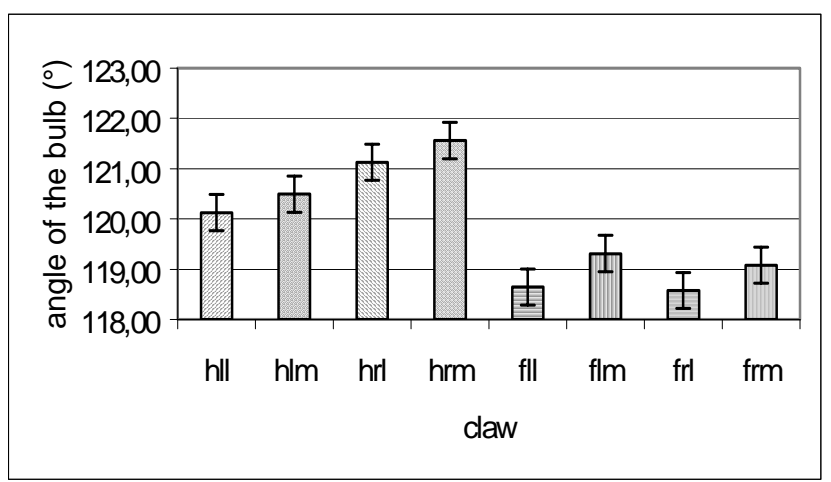

hll - hind left lateral / hind left medial / hrl - hind right lateral / hrm - hind right medial fll - fore left lateral / flm - fore left medial / frl - fore right lateral / frm -fore right medial

Fig. 3: Differences in the angle of the bulb (Unterschiede in der Winkelung des Ballens)

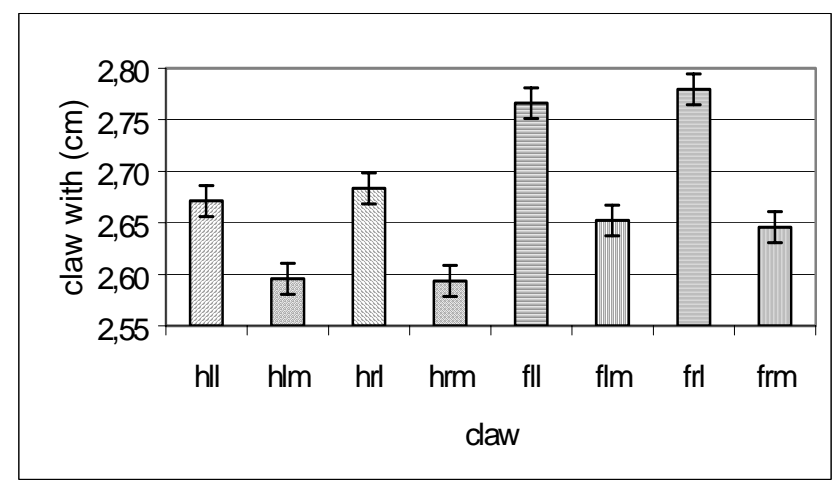

hll - hind left lateral / hind left medial / hrl - hind right lateral / hrm - hind right medial

fll - fore left lateral / flm - fore left medial / frl - fore right lateral / frm -fore right medial

Fig. 4: Differences in claw width (Unterschiede in der Klauenbreite)

\subsection{Measurement number and horn growth}

Depending on the measuring number, horn growth is already significant within the three weeks intervals (not shown) and within the whole study (twelve weeks, Table 2).

Table 2

Range of horn growth in all parameters for the whole study period (Difference and Growth/Month were calculated from Least Squares Means of Measurement 1 and 4) (Hornwachstum aller Parameter innerhalb der gesamten Studie)

\begin{tabular}{|c|c|c|c|c|c|c|c|c|c|}
\hline & $\begin{array}{l}\text { Wall } \\
\text { length } \\
(\mathrm{cm})\end{array}$ & $\begin{array}{l}\text { Bulb } \\
\text { depth } \\
(\mathrm{cm})\end{array}$ & $\begin{array}{l}\text { Bulb } \\
\text { length } \\
(\mathrm{cm})\end{array}$ & $\begin{array}{l}\text { Diagonal } \\
(\mathrm{cm})\end{array}$ & $\begin{array}{l}\text { Sole } \\
\text { length } \\
(\mathrm{cm})\end{array}$ & $\begin{array}{l}\text { Axial } \\
\text { wall } \\
\text { length } \\
(\mathrm{cm})\end{array}$ & $\begin{array}{l}\text { Claw } \\
\text { width } \\
(\mathrm{cm})\end{array}$ & $\begin{array}{l}\text { Dorsal } \\
\text { angle }\left(^{\circ}\right)\end{array}$ & $\begin{array}{l}\text { Bulb } \\
\text { angle }\left(^{\circ}\right)\end{array}$ \\
\hline Measurem. 1 & $\begin{array}{l}4.33 \\
\pm 0.01\end{array}$ & $\begin{array}{l}3.08 \\
\pm 0.01\end{array}$ & $\begin{array}{l}3.02 \\
\pm 0.02\end{array}$ & $\begin{array}{l}5.98 \\
\pm 0.01\end{array}$ & $\begin{array}{l}3.97 \\
\pm 0.02\end{array}$ & $\begin{array}{l}1.27 \\
\pm 0.02\end{array}$ & $\begin{array}{l}2.50 \\
\pm 0.01\end{array}$ & $\begin{array}{l}60.20 \\
\pm 0.16\end{array}$ & $\begin{array}{l}119.15 \pm \\
0.26\end{array}$ \\
\hline Measurem. 4 & $\begin{array}{l}4.55 \\
\pm 0.01\end{array}$ & $\begin{array}{l}3.10 \\
\pm 0.01\end{array}$ & $\begin{array}{l}2.5 \\
\pm 0.02\end{array}$ & $\begin{array}{l}6.62 \\
\pm 0.01\end{array}$ & $\begin{array}{l}5.53 \\
\pm 0.02\end{array}$ & $\begin{array}{l}1.46 \\
\pm 0.02\end{array}$ & $\begin{array}{l}2.85 \\
\pm 0.01\end{array}$ & $\begin{array}{l}57.78 \\
\pm 0.17\end{array}$ & $\begin{array}{l}121.18 \pm \\
0.28\end{array}$ \\
\hline Difference & 0.22 & 0.02 & -0.52 & 0.64 & 1.56 & 0.19 & 0.35 & -2.42 & 2.03 \\
\hline $\begin{array}{l}\text { Growth/ } \\
\text { Month }\end{array}$ & 0.10 & 0.009 & -0.24 & 0.29 & 0.73 & 0.09 & 0.16 & -1.14 & 0.95 \\
\hline
\end{tabular}


The largest growth rate per month was observed in the length of the sole with $0.73 \mathrm{~cm}$, followed by the length of the diagonal $(0.29 \mathrm{~cm})$, claw width $(0.16 \mathrm{~cm})$ and wall length $(0.10 \mathrm{~cm})$ (Table 2).

Bulb length shows a loss of $0.52 \mathrm{~cm}$ within the whole period of investigation. In the same period, the angle of the dorsal wall losses $2.42^{\circ}$ and the angle of the bulb gains $2.03^{\circ}$. This shows that the outer form of the claws changes with increasing weight of the calves and with beginning activity. At start of the study, the claws are steeper than at the last measurement. Within this interval, the calves nearly double their birthweight (body weight in male calves changes from $48.75 \mathrm{~kg}$ to $84.15 \mathrm{~kg}$, females start with $45.10 \mathrm{~kg}$ and reach $74.72 \mathrm{~kg}$ ).

\subsection{The effect of gender}

Male calves show significantly higher values than females in all measured parameters except bulb length, axial wall length and angle of the bulb (Table 3).

Table 3

Horn parameters in male and female calves (Least Squares Mean \pm Standard Error of Estimation*) (Hornwachstum nach Geschlechtern)

\begin{tabular}{llllllllll}
\hline & $\begin{array}{l}\text { Wall } \\
\text { length } \\
(\mathrm{cm})\end{array}$ & $\begin{array}{l}\text { Bulb } \\
\text { depth } \\
(\mathrm{cm})\end{array}$ & $\begin{array}{l}\text { Bulb } \\
\text { length } \\
(\mathrm{cm})\end{array}$ & $\begin{array}{l}\text { Diagonal } \\
(\mathrm{cm})\end{array}$ & $\begin{array}{l}\text { Sole } \\
\text { length } \\
(\mathrm{cm})\end{array}$ & $\begin{array}{l}\text { Axial } \\
\text { wall } \\
\text { length } \\
(\mathrm{cm})\end{array}$ & $\begin{array}{l}\text { Claw } \\
\text { width } \\
(\mathrm{cm})\end{array}$ & $\begin{array}{l}\text { Dorsal } \\
\text { angle }\left(^{\circ}\right)\end{array}$ & $\begin{array}{l}\text { Bulb } \\
\text { angle }\left(^{\circ}\right)\end{array}$ \\
\hline Male & 4.45 & 3.11 & 2.60 & 6.39 & 5.02 & 1.37 & 2.74 & 59.93 & 119.82 \\
& $\pm 0.01^{\mathrm{a}}$ & $\pm 0.01^{\mathrm{a}}$ & \pm 0.01 & $\pm 0.01^{\mathrm{a}}$ & $\pm 0.02^{\mathrm{a}}$ & \pm 0.02 & $\pm 0.01^{\mathrm{a}}$ & $\pm 0.14^{\mathrm{a}}$ & \pm 0.24 \\
Female & 4.39 & 3.06 & 2.62 & 6.21 & 4.83 & 1.32 & 2.59 & 58.58 & 119.91 \\
& $\pm 0.01^{\mathrm{b}}$ & $\pm 0.01^{\mathrm{b}}$ & \pm 0.01 & $\pm 0.01^{\mathrm{b}}$ & $\pm 0.01^{\mathrm{b}}$ & \pm 0.01 & $\pm 0.01^{\mathrm{b}}$ & $\pm 0.11^{\mathrm{b}}$ & \pm 0.19 \\
\hline
\end{tabular}

*Least Squares Means with different letters are significantly different $(\mathrm{p} \leq 0.05)$.

\subsection{The effect of breeding lines}

Fleckvieh (FV) or crossbreds with Fleckvieh Sire (FV-GH) reach higher values in bulb depth and bulb length, diagonal, claw width and angle of the dorsal wall (Table 4).

Table 4

Horn parameters in different breeding lines (Least Squares Mean \pm Standard Error of Estimation*) (Hornwachstum nach Zuchtlinien)

\begin{tabular}{llllllllll}
\hline & $\begin{array}{l}\text { Wall } \\
\text { length } \\
(\mathrm{cm})\end{array}$ & $\begin{array}{l}\text { Bulb } \\
\text { depth } \\
(\mathrm{cm})\end{array}$ & $\begin{array}{l}\text { Bulb } \\
\text { length } \\
(\mathrm{cm})\end{array}$ & $\begin{array}{l}\text { Diagonal } \\
(\mathrm{cm})\end{array}$ & $\begin{array}{l}\text { Sole } \\
\text { length } \\
(\mathrm{cm})\end{array}$ & $\begin{array}{l}\text { Axial } \\
\text { wall } \\
\text { length } \\
(\mathrm{cm})\end{array}$ & $\begin{array}{l}\text { Claw } \\
\text { width } \\
(\mathrm{cm})\end{array}$ & $\begin{array}{l}\text { Dorsal } \\
\text { angle }\left(^{\circ}\right)\end{array}$ & $\begin{array}{l}\text { Bulb angle } \\
\left(^{\circ}\right)\end{array}$ \\
\hline German & 4.43 & 3.05 & 2.55 & 6.21 & 5.00 & 1.27 & 2.64 & 57.62 & 120.15 \\
Holstein & $\pm 0.01^{\mathrm{ab}}$ & $\pm 0.01^{\mathrm{a}}$ & $\pm 0.02^{\mathrm{a}}$ & $\pm 0.01^{\mathrm{a}}$ & $\pm 0.03^{\mathrm{a}}$ & $\pm 0.02^{\mathrm{a}}$ & $\pm 0.01^{\mathrm{a}}$ & $\pm 0.19^{\mathrm{a}}$ & $\pm 0.31^{\mathrm{ab}}$ \\
G-Holstein & 4.44 & 3.05 & 2.53 & 6.27 & 4.93 & 1.50 & 2.63 & 58.90 & 119.51 \\
Fleckvieh & $\pm 0.01^{\mathrm{a}}$ & $\pm 0.01^{\mathrm{a}}$ & $\pm 0.02^{\mathrm{a}}$ & $\pm 0.01^{\mathrm{b}}$ & $\pm 0.02^{\mathrm{b}}$ & $\pm 0.02^{\mathrm{b}}$ & $\pm 0.01^{\mathrm{a}}$ & $\pm 0.15^{\mathrm{b}}$ & $\pm 0.26^{\mathrm{bc}}$ \\
Fleckvieh & 4.39 & 3.09 & 2.66 & 6.35 & 4.85 & 1.23 & 2.71 & 60.42 & 119.41 \\
& $\pm 0.01^{\mathrm{c}}$ & $\pm 0.01^{\mathrm{b}}$ & $\pm 0.02^{\mathrm{b}}$ & $\pm 0.01^{\mathrm{c}}$ & $\pm 0.02^{\mathrm{c}}$ & $\pm 0.02^{\mathrm{a}}$ & $\pm 0.01^{\mathrm{b}}$ & $\pm 0.16^{\mathrm{c}}$ & $\pm 0.26 \mathrm{c}$ \\
Fleckvieh x & 4.41 & 3.14 & 2.69 & 6.37 & 4.92 & 1.38 & 2.70 & 60.10 & 120.38 \\
G-Holstein & $\pm 0.01^{\mathrm{bc}}$ & $\pm 0.01^{\mathrm{c}}$ & $\pm 0.02^{\mathrm{b}}$ & $\pm 0.01^{\mathrm{c}}$ & $\pm 0.02^{\mathrm{b}}$ & $\pm 0.02^{\mathrm{c}}$ & $\pm 0.01^{\mathrm{b}}$ & $\pm 0.18^{\mathrm{c}}$ & $\pm 0.30^{\mathrm{a}}$
\end{tabular}

*Least Squares Means with different letters are significantly different ( $\mathrm{p} \leq 0.05)$.

Sole length in GH is significantly larger than in all other breeding lines, while both crossbred lines show higher values than FV. A heterosis effect of about $13 \%$ seems 
likely for the axial wall length. Both crossbred lines have higher values in axial wall length than the pure lines. GH and FV do not differ significantly in this parameter.

There is a significant breeding line $\mathrm{x}$ gender interaction for sole length within the crossbred lines. Male GH-FV-calves have a significantly longer sole than FV-GHcalves, while female crossbred calves show an inverse pattern (Fig. 5).

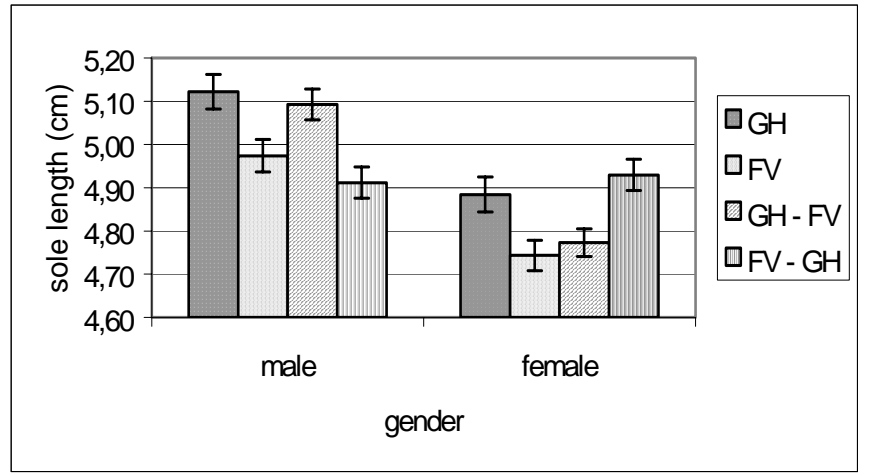

Fig. 5: Sole length in different breeding lines and genders (Sohlenlänge bei verschiedenen Zuchtlinien und Geschlechtern)

There is also an interaction within gender and breeding line for axial wall length (Fig. 6). Within pure German Holsteins, female calves have significantly higher values than males and even female FV.

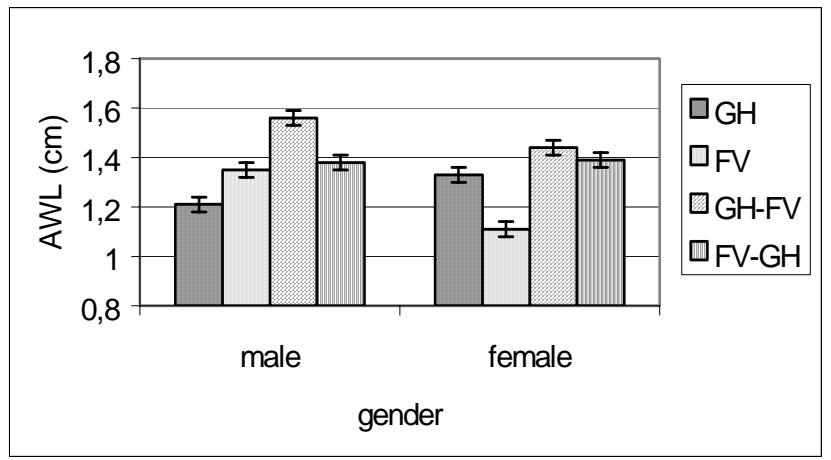

Fig. 6: Axial wall length in different breeding lines and genders (Länge der axialen Sohlenwand bei verschiedenen Zuchtlinien und Geschlechtern)

Calves originating from multiple births had lower values in most of the investigated parameters, but showed a larger axial wall length and larger angles (Table 5). Mean body weight of these calves was $56.04 \mathrm{~kg}$ compared to $66.37 \mathrm{~kg}$ in monoparous calves.

Table 5

Horn parameters in monoparous and pluriparous calves (Least Squares Mean \pm Standard Error of Estimation*) (Hornwachstum bei Einlings- und Zwillingskälbern)

\begin{tabular}{|c|c|c|c|c|c|c|c|c|c|}
\hline & $\begin{array}{l}\text { Wall } \\
\text { length } \\
(\mathrm{cm})\end{array}$ & $\begin{array}{l}\text { Bulb } \\
\text { depth } \\
(\mathrm{cm})\end{array}$ & $\begin{array}{l}\text { Bulb } \\
\text { length } \\
(\mathrm{cm})\end{array}$ & $\begin{array}{l}\text { Diagonal } \\
(\mathrm{cm})\end{array}$ & $\begin{array}{l}\text { Sole } \\
\text { length } \\
(\mathrm{cm})\end{array}$ & $\begin{array}{l}\text { Axial } \\
\text { wall } \\
\text { length } \\
(\mathrm{cm})\end{array}$ & $\begin{array}{l}\text { Claw } \\
\text { width } \\
(\mathrm{cm})\end{array}$ & $\begin{array}{l}\text { Dorsal } \\
\text { angle }\left({ }^{\circ}\right)\end{array}$ & $\begin{array}{l}\text { Bulb } \\
\text { angle }\left(^{\circ}\right)\end{array}$ \\
\hline $\begin{array}{l}\text { Monoparous } \\
\text { calves }\end{array}$ & $\begin{array}{l}4.56 \\
\pm 0.01^{\mathrm{a}}\end{array}$ & $\begin{array}{l}3.22 \\
\pm 0.01^{\mathrm{a}}\end{array}$ & $\begin{array}{l}2.73 \\
\pm 0.01^{\mathrm{a}}\end{array}$ & $\begin{array}{l}6.49 \\
\pm 0.01^{\mathrm{a}}\end{array}$ & $\begin{array}{l}5.12 \\
\pm 0.02^{\mathrm{a}}\end{array}$ & $\begin{array}{l}1.25 \\
\pm 0.01^{\mathrm{a}}\end{array}$ & $\begin{array}{l}2.80 \\
\pm 0.01^{\mathrm{a}}\end{array}$ & $\begin{array}{l}58.72 \\
\pm 0.12^{\mathrm{a}}\end{array}$ & $\begin{array}{l}118.33 \\
\pm 0.20^{\mathrm{a}}\end{array}$ \\
\hline $\begin{array}{l}\text { Pluriparous } \\
\text { calves }\end{array}$ & $\begin{array}{l}4.42 \\
\pm 0.01^{\mathrm{b}}\end{array}$ & $\begin{array}{l}2.94 \\
\pm 0.01^{b}\end{array}$ & $\begin{array}{l}2.48 \\
\pm 0.02^{b}\end{array}$ & $\begin{array}{l}6.11 \\
\pm 0.01^{b}\end{array}$ & $\begin{array}{l}4.73 \\
\pm 0.03^{b}\end{array}$ & $\begin{array}{l}1.44 \\
\pm 0.02^{b}\end{array}$ & $\begin{array}{l}2.54 \\
\pm 0.01^{b}\end{array}$ & $\begin{array}{l}59.80 \\
\pm 0.19^{b}\end{array}$ & $\begin{array}{l}121.39 \\
\pm 0.32^{b}\end{array}$ \\
\hline
\end{tabular}

*Least Squares Means with different letters are significantly different ( $\mathrm{p} \leq 0.05)$. 
3.5. Effect of the lactation number of the mother

Depending on the lactation number of the mother, Table 6 shows the differences for all parameters including body weight.

Table 6

Measuring parameters depending on the lactation number (Least Squares Mean \pm Standard Error of Estimation*) (Klauenparameter in Abhängigkeit zur Laktationsnummer der Mutterkuh)

$\begin{array}{lllllll}\text { Lactation } & 1 & 2 & 3 & 4 & 5 & 6\end{array}$

Parameter

\begin{tabular}{|c|c|c|c|c|c|c|}
\hline $\begin{array}{l}\text { Wall length } \\
(\mathrm{cm})\end{array}$ & $\begin{array}{l}4.3453 \\
\pm 0.0144^{\mathrm{a}}\end{array}$ & $\begin{array}{l}4.4282 \\
\pm 0.0110^{b}\end{array}$ & $\begin{array}{l}4.3536 \\
\pm 0.0159^{\mathrm{a}}\end{array}$ & $\begin{array}{l}4.4405 \\
\pm 0.0167^{b}\end{array}$ & $\begin{array}{l}4.4069 \\
\pm 0.0171^{\mathrm{b}}\end{array}$ & $\begin{array}{l}4.568 \\
\pm 0.0374^{\mathrm{C}}\end{array}$ \\
\hline $\begin{array}{l}\text { Bulb depth } \\
\text { (cm) }\end{array}$ & $\begin{array}{l}3.0285 \\
\pm 0.0165^{\mathrm{a}}\end{array}$ & $\begin{array}{l}3.0568 \\
\pm 0.0126^{a}\end{array}$ & $\begin{array}{l}3.0567 \\
\pm 0.0182^{\mathrm{a}}\end{array}$ & $\begin{array}{l}3.1239 \\
\pm 0.0191^{\mathrm{b}}\end{array}$ & $\begin{array}{l}3.0624 \\
\pm 0.0196^{\mathrm{a}}\end{array}$ & $\begin{array}{l}3.1940 \\
\pm 0.0428^{b}\end{array}$ \\
\hline $\begin{array}{l}\text { Bulb length } \\
\text { (cm) }\end{array}$ & $\begin{array}{l}2.5777 \\
\pm 0.0242^{\mathrm{a}}\end{array}$ & $\begin{array}{l}2.7034 \\
\pm 0.0184^{\mathrm{b}}\end{array}$ & $\begin{array}{l}2.6758 \\
\pm 0.0266^{b}\end{array}$ & $\begin{array}{l}2.6951 \\
\pm 0.0280^{b}\end{array}$ & $\begin{array}{l}2.7131 \\
\pm 0.0287^{b}\end{array}$ & $\begin{array}{l}2.3170 \\
\pm 0.0626^{c}\end{array}$ \\
\hline $\begin{array}{l}\text { Diagonal } \\
\text { (cm) }\end{array}$ & $\begin{array}{l}6.1826 \\
\pm 0.0192^{a}\end{array}$ & $\begin{array}{l}6.2744 \\
\pm 0.0145^{b}\end{array}$ & $\begin{array}{l}6.1821 \\
\pm 0.0210^{a}\end{array}$ & $\begin{array}{l}6.4103 \\
\pm 0.0221^{c}\end{array}$ & $\begin{array}{l}6.2084 \\
\pm 0.0227^{\text {a }}\end{array}$ & $\begin{array}{l}6.5735 \\
\pm 0.0496^{d}\end{array}$ \\
\hline $\begin{array}{l}\text { Sole length } \\
\text { (cm) }\end{array}$ & $\begin{array}{l}4.7841 \\
\pm 0.0305^{\mathrm{ab}}\end{array}$ & $\begin{array}{l}4.7868 \\
\pm 0.0232^{a b}\end{array}$ & $\begin{array}{l}4.7759 \\
\pm 0.0335^{a b}\end{array}$ & $\begin{array}{l}4.7361 \\
\pm 0.0353^{a}\end{array}$ & $\begin{array}{l}4.8419 \\
\pm 0.0362^{b}\end{array}$ & $\begin{array}{l}5.6483 \\
\pm 0.0789^{c}\end{array}$ \\
\hline $\begin{array}{l}\text { Axial wall } \\
\text { length (cm) }\end{array}$ & $\begin{array}{l}1.2787 \\
\pm 0.0251^{\mathrm{bc}}\end{array}$ & $\begin{array}{l}1.3645 \\
\pm 0.0190^{\mathrm{d}}\end{array}$ & $\begin{array}{l}1.3153 \\
\pm 0.0276^{\text {cd }}\end{array}$ & $\begin{array}{l}1.6393 \\
\pm 0.0290^{\text {a }}\end{array}$ & $\begin{array}{l}1.2105 \\
\pm 0.0297^{\mathrm{b}}\end{array}$ & $\begin{array}{l}1.2950 \\
\pm 0.0649 \text { bcd }\end{array}$ \\
\hline $\begin{array}{l}\text { Claw width } \\
(\mathrm{cm})\end{array}$ & $\begin{array}{l}2.6191 \\
\pm 0.0117^{d}\end{array}$ & $\begin{array}{l}2.6589 \\
\pm 0.0088^{b}\end{array}$ & $\begin{array}{l}2.6262 \\
\pm 0.0128\end{array}$ & $\begin{array}{l}2.5716 \\
\pm 0.0135^{c}\end{array}$ & $\begin{array}{l}2.6600 \\
\pm 0.0138^{b}\end{array}$ & $\begin{array}{l}2.9046 \\
\pm 0.0302^{a}\end{array}$ \\
\hline $\begin{array}{l}\text { Wall angle } \\
\left({ }^{\circ}\right)\end{array}$ & $\begin{array}{l}59.328 \\
\pm 0.189 \text { bс }\end{array}$ & $\begin{array}{l}58.877 \\
\pm 0.143^{a}\end{array}$ & $\begin{array}{l}59.366 \\
\pm 0.207^{\text {bc }}\end{array}$ & $\begin{array}{l}59.091 \\
\pm 0.218^{\text {ac }}\end{array}$ & $\begin{array}{l}59.846 \\
\pm 0.224^{b}\end{array}$ & $\begin{array}{l}59.076 \\
\pm 0.488^{\mathrm{ab}}\end{array}$ \\
\hline $\begin{array}{l}\text { Bulb angle } \\
\left({ }^{\circ}\right)\end{array}$ & $\begin{array}{l}121.451 \\
\pm 0.315^{\mathrm{a}}\end{array}$ & $\begin{array}{l}119.755 \\
\pm 0.239^{b}\end{array}$ & $\begin{array}{l}120.149 \\
\pm 0.346^{b}\end{array}$ & $\begin{array}{l}120.275 \\
\pm 0.364^{b}\end{array}$ & $\begin{array}{l}119.820 \\
\pm 0.374^{b}\end{array}$ & $\begin{array}{l}117.743 \\
\pm 0.815^{c}\end{array}$ \\
\hline $\begin{array}{l}\text { Body weight } \\
\text { (kg) }\end{array}$ & $\begin{array}{l}56.803 \\
\pm 0.410^{b}\end{array}$ & $\begin{array}{l}59.458 \\
\pm 0.312^{c}\end{array}$ & $\begin{array}{l}59.061 \\
\pm 0.451^{c}\end{array}$ & $\begin{array}{l}63.208 \\
\pm 0.474^{\mathrm{d}}\end{array}$ & $\begin{array}{l}59.966 \\
\pm 0.487^{c}\end{array}$ & $\begin{array}{l}68.761 \\
\pm 1.062^{\text {a }}\end{array}$ \\
\hline
\end{tabular}

*Least Squares Means with different letters are significantly different $(\mathrm{p} \leq 0.05)$

Calves of older cows exceed those of younger cows in all parameters with exception of the angle of the bulb and axial wall length. This may depend on a higher body weight, but in former results no clear correlation between higher body weight and all other parameters was found. According to the present results, older cows produce calves with larger claws than calves of younger cows.

\section{Discussion and Conclusion}

Linear measurement parameters allow a statement on the size of a claw. The assumption is that having bigger and steeper horn capsules would implicate higher amounts of mineralized and soft tissue inside the claw enabling the claw to burden a higher load.

Wall length was longer in hind than in fore claws. A new aspect is that in all four pairs of claws the medial claws were longer than the lateral claws. In adult cattle, the hind lateral claws and the fore medial claws are longer. This is assumed to be a result of permanent overloading in the normal walking process. For newborn calves, this assumption seems not to be correct. Comparing the medial and lateral hind and fore claws, a similar shape can be found for the first three months of life. 
The rules of cattle footcare and claw trimming described by TOUISSAINT RAVEN (1989) propose a control of the bottom surface of the sole. In a normal sole, the axial part gently slopes beginning "somewhere in the posterior half of the claw and becoming steeper in the interdigital space”. This part of the sole has to be restored in claw trimming. Up to now it is not known, how long the axial wall before the slope naturally is. Therefore we added the axial wall length as a parameter of investigation. By measuring the axial wall length, a judgment of the claw towards soundness could become possible. In this investigation, significant differences between genders and within breeding lines were found. Follow up investigations considering diseases of the claws will study, whether there are significant relations to the axial wall length concerning claw health or not. We propose to add this parameter in future studies including linear measurements.

The ratio between wall length and bulb length is one of the most often used parameters to describe claw quality (UTZ, 1998). Ideal relations should be at 2: 1 . The own results yielded a quotient of 1.41: 1 at the first measurement. At measurement 4 , the quotient is 1.82: 1. This shows that the form of the claw in new-born and growing calves changes rapidly leading to the later seen relations in adult cattle. Perhaps it would be better not to talk of horn growth but of horn development. Since in deed, it is not real growth but a change in claw angles leading to a new shape as described above. These changes are combined with higher body weight and movement leading to the rapid development in claw parameters.

There are large differences in claw parameters depending on the claws themselves comparing fore or hind, medial or lateral claws. As stated above, there are differences between genders, among breeding lines, and even within breeding lines and genders. Thus, claw development can not be described in general. Breeding line and gender must be considered in programs concerning breeding or selection of lines. DISTL (1999) used similar measurement parameters as in the own study for improvement of foot and leg soundness in dairy cattle. He also concluded to consider both genders in the improvement of foot and leg traits.

Considering the interactions between genders and breeding lines, the differences between all measured parameters should lead to a new strategy for progeny testing in breeding programs. Testing a large number of male calves may have the desired effect on the next generation males but inverse effects on females within the same population (and also the inverse way testing only females).

PAULUS and NUSS (2002) collected data of twenty hind digits of 18 months old bulls. They found differences in the length of medial and lateral claws and assumed this to be an anatomical phenomenon. The own results confirm this for calves. Up to now no clear results are available for adult female cattle.

The age of the mother has a strong influence on the calf's claw development. This aspect underlines the importance of life-production. If older cows produce calves with improved claw development assuming that would lead to better claw health, breeding strategies should concern this aspect besides production parameters.

Nevertheless, the central factor in describing claw development is, that up to now it is not really clear, which parameter is ideal for selection and health evaluation. There are no strait results which would generally confirm relations between single parameters and diseases or disorders of the claw. 
Though high efforts have been done to develop improved trimming methods, stronger activities should be aimed at programs analyzing data on measurable parameters, which fit better the needs of a more scientific knowledge based diagnosis of claw and leg problems.

\section{References}

BAUMGARTNER, C.:

Untersuchung über Klauenmaße als Hilfsmerkmale für die Selektion auf Klauengesundheit an Töchtergruppen von Deutschen Fleckviehbullen. Diss. Med. vet. München, 1988 DISTL, O.:

Zucht auf ein gesundes Fundament beim Milchrind. (Breeding for soundness of feet and legs in dairy HUBER, M.: cattle). Züchtungskunde 71 (1999), 446 - 458

Untersuchungen über Klauenparameter an Jungbullen in den Bayerischen Eigenleistungsprüfungsanstalten. Diss. Med vet., München, 1983

HUBER, J.:

Klauenerkrankungen bei Milchkühen in verschiedenen Haltungsformen - im Vergleich Anbindehaltung und Laufstallhaltung. Diss. Med. vet., Wien, 2002

LANDERER, R.; LISCHER, C.; GEYER, H.; WEHRLE, M.; HOFSTETTER, H.:

Hoof shape and horn growth/wear in relation to foot lesions in Brown Swiss cattle on alpine pasture, in Proceedings. $10^{\text {th }}$ Int Symp Lameness in Ruminants 1998, 53-55

LIVESEY, C.T.; JOHNSTON, A.M.; MARSH, C.; MAY, S.A.; METCALF, J.A.:

Hoof horn growth in Holstein cattle from first calving until six weeks after second calving, in Proceedings. $10^{\text {th }}$ Int Symp Lameness in Ruminants 1998, 51-52

MANSKE, T.:

Hoof lesions and lameness in Swedish dairy cattle. Thesis, Swedish University of Agricultural Sciences, Skara, 2002

MEYER, K.; GALBRAITH, H.:

Claw dimensions and rates of hoof growth and wear in Holstein dairy cows before and after turnout to pasture from concrete cubicles and straw yards, in Proceedings. $10^{\text {th }}$ Int Symp Lameness in Ruminants 1998, 49-50

MÜLLER, H.W.; SPINDLER, G.; SCHLEITER, H.:

Messungen an Klauen von auf Vollspaltenböden gehaltenen Mastkälbern. Mh. Vet. Med. 31 (1975), 693-696

NITSCHE, W.:

Untersuchungen über das Wachstum und die Abnutzung des Klauenhornes bei Mastkälbern unter unterschiedlichen Aufstallungsvarianten. Chir. Vet. 11 (1974), 52-53

PAULUS, N.; NUSS, K.:

Claw measures at defined sole thickness, in Proceedings. $12^{\text {th }}$ Int Symp Lameness in Ruminants 2002, 428-430

REURINK, A.; VAN ARENDONK, J.:

Relationship of claw disorders and claw measurements with efficiency of production in dairy cattle. $38^{\text {th }}$ Meeting of the European Association for Animal Production, Lisbon, Portugal, 1987

RUSSKE, A.K.:

Entwicklung von Klauenform und Klauenhorneigenschaften bei Jungrindern der Rassen Deutsche Holsteins und Deutsches Braunvieh. Diss. Med. vet., Hannover, 2001

SAS / STAT

User's Guide Volume 2, GLM-VARCOMP, Version 6 Fourth Edition, by SAS Institute Inc., Cary, NC, USA, 1993, 891-996

TOUSSAINT RAVEN, E.:

UTZ, J.:

Cattle footcare and claw trimming. Ipswich, UK: Farming Press, 1989

Exterieurbeurteilung Rind. In: BREM G., ed. Exterieurbeurteilung landwirtschaftlicher Nutztiere. Stuttgart, Germany, Eugen Ulmer Verlag, 1998, 180-181

VERMUNT, J.J.; GREENOUGH, P.R.:

Structural characteristics of the bovine claw: Horn growth and wear, horn hardness and claw conformation. Br. Vet. J. 151 (1995), 157-180 
VAN DER TOL, P.P.J.; METZ, J.H.M.; NOORDHUIZEN-STASSEN, E.M.; BACK, W.; BRAAM, C.R.; WEIJS, W.A.:

Pressure distribution on the bovine claw while standing, in Proceedings. $12^{\text {th }}$ Int Symp Lameness in Ruminants 2002; 202-205.

Received: 2003-07-03

Accepted: 2003-09-16

Author's addresses

Dr. STEFAN NÜSKE

Lehr- und Versuchsgut der Tierärztlichen Fakultät der Ludwig-Maximilians-Universität

Hubertusstr. 12

D-85764 Oberschleissheim

Priv. Doz. Dr. Dr. habil. ARMIN M. SCHOLZ

Lehr- und Versuchsgut der Tierärztlichen Fakultät der Ludwig-Maximilians-Universität

Hubertusstr. 12

D-85764 Oberschleissheim

Univ.-Prof. Dr. Dr. habil. MARTIN FÖRSTER

Institut für Tierzucht und Allgemeine Landwirtschaftslehre der Ludwig-Maximilians-Universität

Veterinärstr. 13

D-80805 München 